This is an electronic reprint of the original article. This reprint may differ from the original in pagination and typographic detail.

Author(s): Varguinin, A.; Silaev, Mikhail

Title: Self-consistent calculation of the flux-flow conductivity in diffusive superconductors

Year: $\quad 2017$

Version:

Please cite the original version:

Varguinin, A., \& Silaev, M. (2017). Self-consistent calculation of the flux-flow conductivity in diffusive superconductors. Physical Review B, 96(21), Article 214507. https://doi.org/10.1103/PhysRevB.96.214507

All material supplied via JYX is protected by copyright and other intellectual property rights, and duplication or sale of all or part of any of the repository collections is not permitted, except that material may be duplicated by you for your research use or educational purposes in electronic or print form. You must obtain permission for any other use. Electronic or print copies may not be offered, whether for sale or otherwise to anyone who is not an authorised user. 


\title{
Self-consistent calculation of the flux-flow conductivity in diffusive superconductors
}

\author{
A. Vargunin ${ }^{1,2}$ and M. A. Silaev ${ }^{3}$ \\ ${ }^{1}$ Department of Theoretical Physics, The Royal Institute of Technology, SE-10691 Stockholm, Sweden \\ ${ }^{2}$ Institute of Physics, University of Tartu, EE-50411 Tartu, Estonia \\ ${ }^{3}$ Department of Physics and Nanoscience Center, University of Jyväskylä, P.O. Box 35 (YFL), FI-40014 University of Jyväskylä, Finland
}

(Received 5 September 2017; revised manuscript received 18 November 2017; published 18 December 2017)

\begin{abstract}
In the framework of Keldysh-Usadel kinetic theory, we study the temperature dependence of flux-flow conductivity (FFC) in diffusive superconductors. By using self-consistent vortex solutions we find the exact values of dimensionless parameters that determine the diffusion-controlled FFC both in the limit of the low temperatures and close to the critical one. Taking into account the electron-phonon scattering, we study the transition between flux-flow regimes controlled by either the diffusion or the inelastic relaxation of nonequilibrium quasiparticles. We demonstrate that the inelastic electron-phonon relaxation leads to the strong suppression of FFC compared to the previous estimates, making it possible to obtain numerical agreement with experimental results.
\end{abstract}

DOI: 10.1103/PhysRevB.96.214507

\section{INTRODUCTION}

Vortex motion is an important process that determines resistive properties of type-II superconductors in the flux-flow regime. At magnetic fields $B$ much weaker than the upper critical one $H_{c 2}$, the density of vortex lines is low, and the total electric losses are given by the superposition of the individual vortex contributions. In this regime, the flux-flow resistivity $\rho_{f}$ is proportional to the density of vortex lines, $\rho_{f} \sim B / H_{c 2}$, as described by the general expression suggested by Bardeen and Stephen [1]. The inverse quantity $\sigma_{f}=1 / \rho_{f}$, the flux-flow conductivity (FFC), is therefore given by

$$
\sigma_{f} / \sigma_{n}=\beta H_{c 2} / B
$$

where $\sigma_{n}$ is the normal-state conductivity and $\beta$ is the numerical coefficient which is determined by the particular microscopic model.

For superconducting materials with a high rate of impurity scattering, the numerical value of $\beta \approx 0.9$ at low temperatures has been reported by Gor'kov and Kopnin [2] (GK). This value of the dimensionless parameter was obtained using the approximate vortex solution found by Watts-Tobin and Waterworth [3] and is discussed in detail below. To date the exact value of $\beta$ has been unknown, and it is reported in the present paper based on the fully self-consistent vortex structure calculations.

At elevated temperatures, two different regimes of the vortex motion have been considered, depending on the dominant mechanism of the relaxation [4]. One of them is the diffusion-controlled flux flow when the generation of nonequilibrium quasiparticles near the vortex line is balanced by their diffusion to infinity. As the temperature approaches $T_{c}$, this mechanism results in the divergent behavior of FFC given by [4-6]

$$
\beta \approx \beta_{0}\left(1-T / T_{c}\right)^{-1 / 2},
$$

with temperature-independent $\beta_{0}$. Qualitatively, this behavior is explained by the vortex core size increase proportional to the Ginzburg-Landau coherence length $\xi_{\mathrm{GL}}(T) \sim \sqrt{D /\left(T_{c}-T\right)}$, where $D$ is the diffusion coefficient. This dependence is in qualitative agreement with experimental results [7] pointing to the significant increase of $\beta$ as temperature approaches
$T_{c}$. However, quantitative agreement is lacking. Initially, the value of $\beta_{0} \approx 1.1$ was reported [5], which by coincidence was in good agreement with experiments [7]. However, subsequently, this result was revised to $\beta_{0} \approx 4.04$ by Larkin and Ovchinnikov $[4,6](\mathrm{LO})$ which is several times larger than the measured values in various superconductors [8-14].

When the temperature becomes sufficiently close to $T_{c}$ the relaxation is dominated by the inelastic electron-phonon collisions. This regime is described by generalized timedependent Ginzburg-Landau theory (GTDGL), yielding the FFC decreasing with temperature [4],

$$
\beta \sim\left(T_{c} \tau_{\mathrm{ph}}\right)\left(1-T / T_{c}\right)^{1 / 2},
$$

where $\tau_{\mathrm{ph}}$ is the electron-phonon relaxation time. In the limit $T \rightarrow T_{c}$ the gapless superconducting state is realized. In this case the decrease in $\beta(T)$ saturates at $\beta=1.45$ [15].

The crossover between the two regimes described by Eqs. (2) and (3) occurs at the temperatures $\tau_{\mathrm{ph}}\left(T_{c}-T\right) \sim 1$ when the diffusion rate becomes of the order of the electronphonon relaxation rate, $D \xi_{\mathrm{GL}}^{-2} \sim \tau_{\mathrm{ph}}^{-1}$. That yields an estimation of the maximal value $\max (\beta) \sim \sqrt{T_{c} \tau_{\mathrm{ph}}}$ obtained from Eqs. (2) and (3) at the upper and lower borders of their applicability, respectively.

Although the estimations of $\max (\beta)$ obtained from Eqs. (2) and (3) agree in order of magnitude, the temperature domains where these equations are valid do not overlap. Therefore, to find the behavior of $\beta$ in the transition interval from the diffusion-controlled regime to the GTDGL regime one needs to improve the accuracy of the calculation taking into account both mechanisms of relaxation. This is the problem we address in the present paper. We study the linear-response FFC of the sparse vortex lattices in a small magnetic field by solving numerically kinetic equations describing nonequilibrium states generated by moving isolated vortices. To find kinetic coefficients and driving terms we use vortex structures calculated self-consistently.

For the diffusion-controlled vortex motion, we calculate the temperature dependence $\beta=\beta(T)$ and compare it with the interpolation curve suggested in earlier works $[4,7]$. Taking into account the electron-phonon scattering, we demonstrate that it leads to significant suppression of the FFC at intermediate 
temperatures, $\tau_{\mathrm{ph}}\left(T_{c}-T\right) \sim 1$, compared to the estimations obtained from Eqs. (2) and (3). Using the electron-phonon relaxation rate $\tau_{\mathrm{ph}}^{-1}$ as the fitting parameter, we obtain numerically accurate fits to the experimentally measured temperature dependencies of FFC in $\mathrm{Zr}_{3} \mathrm{Rh}$ [14] and $\mathrm{Nb}-\mathrm{Ta}$ [8,9].

The structure of this paper is as follows. In Sec. II we introduce the Keldysh-Usadel description of the kinetic processes in dirty superconductors. Here the basic components of the kinetic theory are discussed, including kinetic equations, self-consistency equations for the order parameter, and the general expression for the viscous force acting on moving vortices. Section III introduces the $\theta$ parametrization of the theory. Calculated temperature dependencies of FFC are reported in Sec. IV for different regimes. The diffusioncontrolled flux-flow is discussed in Sec. IV A, and the influence of increasing electron-phonon relaxation rate is studied in Sec. IV B. A summary is given in Sec. V.

\section{KINETIC EQUATIONS AND THE FORCES ACTING ON THE MOVING VORTEX LINE}

The quasiclassical Green's function (GF) is defined as

$$
\check{g}=\left(\begin{array}{cc}
\hat{g}^{R} & \hat{g}^{K} \\
0 & \hat{g}^{A}
\end{array}\right),
$$

where $g^{K}$ is the $(2 \times 2$ matrix $)$ Keldysh component and $\hat{g}^{R(A)}$ are the retarded (advanced) ones. The GF $\check{g}=\check{g}\left(t_{1}, t_{2}, \boldsymbol{r}\right)$ depends on times $t_{1,2}$ and a single spatial coordinate $\boldsymbol{r}$. In dirty superconductors $\check{g}$ obeys the Keldysh-Usadel equation

$$
\left\{\hat{\tau}_{3} \partial_{t}, \check{g}\right\}_{t}=D \hat{\partial}_{\mathbf{r}}\left(\check{g} \circ \hat{\partial}_{\mathbf{r}} \check{g}\right)+[\hat{H}, \check{g}]_{t}+\check{I} .
$$

Here $\hat{\tau}_{0,1,2,3}$ are Pauli matrices in Nambu space, $D$ is the diffusion constant, and $\hat{H}(\boldsymbol{r}, t)=i \hat{\Delta}-i e \phi \hat{\tau}_{0}$, where $\hat{\Delta}(t)=$ $i|\Delta| \hat{\tau}_{2} e^{-i \varphi \hat{\tau}_{3}}$ is the gap operator, $\varphi$ is the gap phase, $|\Delta|$ is the gap modulus, and $\phi$ is the electrostatic potential.

In Eq. (5) the commutator is defined as $[X, g]_{t}=$ $X\left(t_{1}\right) g\left(t_{1}, t_{2}\right)-g\left(t_{1}, t_{2}\right) X\left(t_{2}\right)$, with a similar definition for the anticommutator $\{X, g\}_{t}$; in particular, $\left\{\hat{\tau}_{3} \partial_{t}, \check{g}\right\}_{t}=$ $\hat{\tau}_{3} \partial_{t_{1}} \check{g}\left(t_{1}, t_{2}\right)+\partial_{t_{2}} \check{g}\left(t_{1}, t_{2}\right) \hat{\tau}_{3}$. The symbolic product operator is given by $(A \circ B)\left(t_{1}, t_{2}\right)=\int_{-\infty}^{\infty} d t A\left(t_{1}, t\right) B\left(t, t_{2}\right)$, and the covariant differential superoperator is

$$
\hat{\partial}_{\mathbf{r}} \check{g}=\nabla \check{g}-i e\left[\hat{\tau}_{3} A, \check{g}\right]_{t} .
$$

The collision integral in (5) is given by

$$
\check{I}=i(\check{g} \circ \check{\Sigma}-\check{\Sigma} \circ \check{g}),
$$

where the self-energy $\check{\Sigma}$ may contain contributions from different relaxation processes. Here we take into account only the electron-phonon scattering, which plays an important role in the energy relaxation.

The Keldysh-Usadel equation (5) is complemented by the normalization condition $(\check{g} \circ \check{g})\left(t_{1}, t_{2}\right)=\check{\delta}\left(t_{1}-t_{2}\right)$, which allows us to introduce parametrization of the Keldysh component in terms of the distribution function

$$
\begin{gathered}
\hat{g}^{K}\left(t_{1}, t_{2}\right)=\left(\hat{g}^{R} \circ \hat{f}\right)\left(t_{1}, t_{2}\right)-\left(\hat{f} \circ \hat{g}^{A}\right)\left(t_{1}, t_{2}\right), \\
\hat{f}\left(t_{1}, t_{2}\right)=\hat{\tau}_{0} f_{L}\left(t_{1}, t_{2}\right)+\hat{\tau}_{3} f_{T}\left(t_{1}, t_{2}\right) .
\end{gathered}
$$

The deviation of $f_{L}$ from the equilibrium distribution is related to the effective temperature change, and $f_{T}$ is the charge imbalance on the quasiparticle branch.

To proceed we introduce the mixed representation in the time-energy domain as follows: $\breve{g}\left(t_{1}, t_{2}\right)=$ $\int_{-\infty}^{\infty} \check{g}(\varepsilon, t) e^{-i \varepsilon\left(t_{1}-t_{2}\right)} d \varepsilon /(2 \pi)$, where $t=\left(t_{1}+t_{2}\right) / 2$.

The Keldysh-Usadel equation (5) can be simplified by using the gradient approximation. In order to keep the resulting kinetic equations gauge invariant we use the modified GFs $\check{g}_{\text {new }}\left(t_{1}, t_{2}\right)=\hat{W}\left(t_{1}, t\right) \check{g}\left(t_{1}, t_{2}\right) \hat{W}\left(t, t_{2}\right)$, where the link operator is given by $\hat{W}\left(t_{1}, t_{2}\right)=e^{i \hat{\tau}_{3} \int_{t_{1}}^{t_{2}} e \phi d t}$. This transformation leads to the local chemical potential shift. To take this into account we will use the substitution $f_{T}(\varepsilon, t) \rightarrow f_{T}(\varepsilon, t)+e \phi \partial_{\varepsilon} f_{0}$, where $f_{0}(\varepsilon)=\tanh (\varepsilon / 2 T)$ is the equilibrium distribution function. After this transformation $f_{T}(\varepsilon, t)$ denotes the deviation from the local equilibrium distribution.

Then, keeping the first-order nonequilibrium terms, we obtain the system of two coupled kinetic equations that determine both the transverse and longitudinal distribution function components $f_{L, T}=f_{L, T}(\varepsilon, t)$ (a detailed derivation is given in Appendix A):

$$
\begin{aligned}
& \nabla\left(\mathcal{D}_{T} \nabla f_{T}\right)+\boldsymbol{j}_{e} \cdot \nabla f_{L}+2 i \operatorname{Tr}\left[\left(\hat{g}^{R}+\hat{g}^{A}\right) \hat{\Delta}\right] f_{T} \\
& \quad=\partial_{\varepsilon} f_{0} \operatorname{Tr}\left[\hat{\tau}_{3} \hat{\partial}_{t} \hat{\Delta}\left(\hat{g}^{R}+\hat{g}^{A}\right)\right], \\
& \nabla\left(\mathcal{D}_{L} \nabla f_{L}\right)+\boldsymbol{j}_{e} \cdot \nabla f_{T}+2 i \operatorname{Tr}\left[\hat{\tau}_{3}\left(\hat{g}^{R}-\hat{g}^{A}\right) \hat{\Delta}\right] f_{T} \\
& \quad=-\partial_{\varepsilon} f_{0} \operatorname{Tr}\left[\hat{\partial}_{t} \hat{\Delta}\left(\hat{g}^{R}-\hat{g}^{A}\right)\right]-\operatorname{Tr} \hat{J},
\end{aligned}
$$

where the energy-dependent diffusion coefficients $\mathcal{D}_{T, L}$ and the spectral charge current $\boldsymbol{j}_{e}$ are given by

$$
\begin{gathered}
\mathcal{D}_{T}=D \operatorname{Tr}\left(\hat{\tau}_{0}-\hat{\tau}_{3} \hat{g}^{R} \hat{\tau}_{3} \hat{g}^{A}\right), \\
\mathcal{D}_{L}=D \operatorname{Tr}\left(\hat{\tau}_{0}-\hat{g}^{R} \hat{g}^{A}\right), \\
\boldsymbol{j}_{e}=D \operatorname{Tr}\left[\hat{\tau}_{3}\left(\hat{g}^{R} \hat{\nabla} \hat{g}^{R}-\hat{g}^{A} \hat{\nabla} \hat{g}^{A}\right)\right] .
\end{gathered}
$$

In Eqs. (10), (11), and (14) we use the covariant time derivative and spatial gradient defined by $\hat{\partial}_{t}=\hat{\tau}_{0} \partial_{t}+2 i e \phi \hat{\tau}_{3}$ and $\hat{\nabla}=$ $\boldsymbol{\nabla}-\boldsymbol{i} \boldsymbol{e} \boldsymbol{A}\left[\hat{\tau}_{3},\right]$. We omit the driving terms containing electric field, which is justified in type-II superconductors with large Ginzburg-Landau parameters. In such systems the dominating driving terms are those containing order parameter gradients.

The electron-phonon collision integral on the right-hand side of kinetic equation (11) is $\hat{J}=\hat{I}^{K}-\hat{I}^{R} \circ \hat{f}+\hat{f} \circ \hat{I}^{A}$, where the components of $\check{I}$ are given by Eq. (7) with electronphonon self-energies [16]

$$
\begin{aligned}
\hat{\Sigma}^{R / A / K}(\varepsilon) & =-\frac{\lambda_{\mathrm{ph}}}{56 \zeta(3) T_{c}^{2}} \int_{-\infty}^{\infty} d \omega \tilde{\Sigma}^{R / A / K}(\omega, \varepsilon), \\
\tilde{\Sigma}^{R / A}(\omega, \varepsilon) & =D^{K}(\omega) \hat{g}^{R / A}(\varepsilon+\omega)-D^{R / A}(\omega) \hat{g}^{K}(\varepsilon+\omega), \\
\tilde{\Sigma}^{K}(\omega, \varepsilon) & =D^{K}(\omega) \hat{g}^{K}(\varepsilon+\omega)-D^{R A}(\omega) \hat{g}^{R A}(\varepsilon+\omega) .
\end{aligned}
$$

Here

$$
\begin{gathered}
D^{R / A}(\omega)= \pm i \omega|\omega|, \\
D^{K}(\omega)=D^{R A}(\omega) \operatorname{coth}\left(\frac{\omega}{2 T}\right)
\end{gathered}
$$


are the free-phonon propagators, $D^{R A}=D^{R}-D^{A}$ and $\hat{g}^{R A}=\hat{g}^{R}-\hat{g}^{A}$. We parametrize the electron-phonon selfenergy by the dimensionless constant $\lambda_{\mathrm{ph}}=\left(T_{c} \tau_{\mathrm{ph}}\right)^{-1}$, where $\tau_{\mathrm{ph}}$ is the electron-phonon relaxation time at $T=T_{c}$.

The force acting on the moving vortex line from the dissipative environment can be calculated according to the expression $[4,17]$

$$
\boldsymbol{F}_{\text {env }}=v \int d^{2} \boldsymbol{r} \int_{-\infty}^{\infty} \frac{d \varepsilon}{4} \operatorname{Tr}\left(\hat{g}^{\mathrm{nst}} \hat{\partial}_{\boldsymbol{r}} \hat{\Delta}\right),
$$

where $v$ is the density of states and $\hat{g}^{\text {nst }}$ is the nonstationary Green's function, which can be obtained by the gradient expansion as follows:

$$
\begin{aligned}
\hat{g}^{\mathrm{nst}}= & -\frac{i}{2} \hat{\partial}_{t}\left(\hat{g}^{R}+\hat{g}^{A}\right) \partial_{\varepsilon} f_{0} \\
& +\left(\hat{g}^{R}-\hat{g}^{A}\right)\left(f_{L}-f_{0}\right)+\left(\hat{g}^{R} \hat{\tau}_{3}-\hat{\tau}_{3} \hat{g}^{A}\right) f_{T} .
\end{aligned}
$$

Here $f_{T}$ denotes the deviation from the local equilibrium, as discussed above.

In Eq. (18) we neglect the contribution from the normal component of the charge current. This assumption is well justified for the small magnetic fields compared to the upper critical one [18].

\section{III. $\theta$ PARAMETRIZATION}

In general, the normalization condition allows one to parametrize GF by complex variables $\theta$ and $\tilde{\varphi}$. For the axially symmetric vortices the latter coincides with the vortex phase $\tilde{\varphi}=\varphi$. In this case we have

$$
\begin{gathered}
\hat{g}^{R}=\hat{\tau}_{3} \cosh \theta+i \hat{\tau}_{2} e^{-i \hat{\tau}_{3} \varphi} \sinh \theta, \\
\hat{g}^{A}=-\hat{\tau}_{3} \cosh \theta^{*}-i \hat{\tau}_{2} e^{-i \hat{\tau}_{3} \varphi} \sinh \theta^{*} .
\end{gathered}
$$

The complex parameter $\theta=\theta(r)$, depending only on the distance to the vortex center $r$, is given by the solution of the Usadel equation

$$
\nabla_{r}^{2} \theta-\frac{\sinh 2 \theta}{2 r^{2}}+\frac{2 i}{D}\left[\left(\varepsilon+\frac{i}{2 \tau}\right) \sinh \theta-|\Delta| \cosh \theta\right]=0,
$$

where $\nabla_{r}^{2}=\partial_{r}^{2}+r^{-1} \partial_{r}$ (see Appendix C). The boundary conditions for Eq. (22) read

$$
\begin{gathered}
\theta(0)=0, \\
\sinh \theta(\infty)=\Delta_{0} / \sqrt{[\varepsilon+i /(2 \tau)]^{2}-\Delta_{0}^{2}},
\end{gathered}
$$

where $\Delta_{0}=|\Delta(r=\infty)|$. Electron-phonon scattering with characteristic time $\tau$ in Eqs. (22)-(24) regularizes spectral functions near the gap edge singularity. At low temperatures electron-phonon scattering does not affect the calculation results. In the vicinity of $T_{c}$, its value is important since the inelastic relaxation dominates the dissipation. To describe the effects of electron-phonon scattering on the relaxation we calculate $\tau$ self-consistently within the relaxation-time approximation described in Appendix B. In this approach

$$
\frac{1}{\tau}=\frac{\lambda_{\mathrm{ph}} \cosh \frac{\varepsilon}{2 T}}{14 \zeta(3) T_{c}^{2}} \int_{-\infty}^{\infty} \frac{\omega|\omega| d \omega \mathrm{Re} \cosh [\theta(\varepsilon+\omega)]}{\sinh \frac{\omega}{2 T} \cosh \frac{\varepsilon+\omega}{2 T}} .
$$

To determine the gap profile, we use a stationary selfconsistency equation written in the form

$$
|\Delta| \ln \left(T / T_{c}\right)=2 \pi T \sum_{n}\left(\sin \theta_{n}^{M}-|\Delta| / \omega_{n}\right) .
$$

Here the summation runs over Matsubara frequencies $\omega_{n}=$ $(2 n+1) \pi T, n=0, \ldots, \infty$, and the angle $\theta_{n}^{M}(r)$ parametrizes the imaginary-frequency GF obtained by the transformation $\theta \rightarrow-i \theta_{n}^{M}$ from Eqs. (20) and (21) in the upper and lower half planes, respectively. To obtain $\theta_{n}^{M}(r)$ we solve the Usadel equation (22) with $\theta \rightarrow-i \theta_{n}^{M}$ and $\varepsilon \rightarrow i \omega_{n}$. We assume that the condition $\omega_{n} \tau \gg 1$ is always satisfied and neglect the relaxation time correction while solving Eq. (22) for the imaginary frequencies. The boundary conditions read $\theta_{n}^{M}(0)=0$ and

$$
\theta_{n}^{M}(\infty)=\sin ^{-1}\left[\Delta_{0} / \sqrt{\Delta_{0}^{2}+\omega_{n}^{2}}\right] .
$$

The driving terms in kinetic equations (10) and (11) are given by the time derivatives of the order parameter, which for the steady vortex motion can be written as $\partial_{t} \hat{\Delta}=-\boldsymbol{v}_{L} \cdot \nabla \hat{\Delta}$, where $\boldsymbol{v}_{L}$ is the vortex velocity. For the axially symmetric vortex, this form of driving terms allows for the separation of the variables using the ansatz

$$
\begin{gathered}
f_{L}-f_{0}=v_{L} \tilde{f}_{L} \partial_{\varepsilon} f_{0} \cos \varphi, \\
f_{T}=v_{L} \tilde{f}_{T} \partial_{\varepsilon} f_{0} \sin \varphi .
\end{gathered}
$$

Here the amplitudes $\tilde{f}_{L, T}=\tilde{f}_{L, T}(r)$ are defined by Eqs. (10) and (11), which can be written in the compact form as follows:

$$
\begin{aligned}
& \partial_{r}\left(r \mathcal{D}_{T} \partial_{r} \tilde{f}_{T}\right)-\left(\mathcal{D}_{T}-8|\Delta| r^{2} \cosh \vartheta \sin \eta\right) \frac{\tilde{f}_{T}}{r} \\
&=4|\Delta| \cosh \vartheta \sin \eta-\mathcal{D}_{L} \sinh (2 \vartheta) \tan \eta \frac{\tilde{f}_{L}}{r}, \\
& \partial_{r}\left(r \mathcal{D}_{L} \partial_{r} \tilde{f}_{L}\right)-\mathcal{D}_{L} \frac{\tilde{f}_{L}}{r}= \mathcal{D}_{T} \tanh \vartheta \sin (2 \eta) \frac{\tilde{f}_{T}}{r} \\
&-4 r \sinh \vartheta \cos \eta \partial_{r}|\Delta| \\
&+r \tilde{f}_{L} v_{\text {out }}-r j_{\text {in }},
\end{aligned}
$$

where $\vartheta=\operatorname{Re} \theta$ and $\eta=\operatorname{Im} \theta$. For a detailed derivation see Appendix C. The last two terms in Eq. (31) describe scatteringout and scattering-in contributions to the inelastic relaxation of the nonequilibrium longitudinal imbalance. The integrals are given by

$$
\begin{aligned}
v_{\mathrm{out}}= & \frac{2 \lambda_{\mathrm{ph}} \cos \eta}{7 \zeta(3) T_{c}^{2}} \int_{-\infty}^{\infty} d \omega \omega|\omega| \cos [\eta(\varepsilon+\omega)] \\
& \times \cosh [\vartheta(\varepsilon)-\vartheta(\varepsilon+\omega)]\left[1 / f_{0}(\omega)-f_{0}(\varepsilon+\omega)\right],(32) \\
j_{\text {in }}= & \frac{2 \lambda_{\mathrm{ph}} \cos \eta}{7 \zeta(3) T_{c}^{2} \partial_{\varepsilon} f_{0}} \int_{-\infty}^{\infty} d \omega \omega|\omega| \cos [\eta(\varepsilon+\omega)] \tilde{f}_{L}(\varepsilon+\omega) \\
& \times \cosh [\vartheta(\varepsilon)-\vartheta(\varepsilon+\omega)] \partial_{\varepsilon} f_{0}(\varepsilon+\omega)\left[f_{0}(\varepsilon)+1 / f_{0}(\omega)\right] .
\end{aligned}
$$


Kinetic equations (30) and (31) are solved numerically within the interval $0 \leqslant r \leqslant r_{c}$, where $r_{c}$ is the cell radius. For the regime of diffusion-controlled dissipation we choose an interval large enough that the result is not sensitive to $r_{c}$. When discussing the crossover to the inelastic relaxation-driven dissipation, we set the interval to be larger than the inelastic relaxation length $\sqrt{D \tau}$, which determines the decay of $\tilde{f}_{L}$ at large distances. We use the following boundary conditions:

$$
\begin{aligned}
\tilde{f}_{T}(r=0) & =\tilde{f}_{L}(r=0)=0, \\
\tilde{f}_{T}\left(r=r_{c}\right) & =1 / 2 r_{c}, \\
\partial_{r} \tilde{f}_{L}\left(r=r_{c}\right) & =0 .
\end{aligned}
$$

Here the condition at $r=0$ in Eq. (34) follows from the regularity of the solutions at the origin, while the condition at $r_{c}$ provides the disappearance of the charge imbalance and the absence of the heat flow into the bulk.

The viscous friction force acting on an individual moving vortex can be written as $\boldsymbol{F}_{\text {env }}=-\varrho \boldsymbol{v}_{L}$. We present the viscosity coefficient in the form $\varrho=\pi \hbar v(\alpha+\gamma)$, separating the contributions of the driving terms related to the gap modulus and phase gradients (see Appendix C). In general the flux-flow conductivity can be expressed through the vortex viscosity as follows [5]:

$$
\sigma_{f}=\varrho /\left(B \phi_{0}\right),
$$

where $\phi_{0}$ is the magnetic flux quantum. Taking into account the normal-state Drude conductivity, $\sigma_{n}=2 e^{2} v D$, we write the FFC in the form (1) with

$$
\beta=c(\alpha+\gamma) /\left(2 e D H_{c 2}\right) .
$$

The upper critical field $H_{c 2}$ is determined by the Maki equation [19], $\ln \left(T / T_{c}\right)+\psi\left[1 / 2+e H_{c 2} D /(2 \pi c T)\right]=\psi(1 / 2)$, where $\psi$ is the digamma function. The low-temperature limit gives $H_{c 2}=\phi_{0} T_{c} /\left(2 D \gamma_{0}\right)$, where $\gamma_{0}=1.781$. Close to the critical temperature one obtains $H_{c 2}=\phi_{0} /\left(2 \pi \xi_{\mathrm{GL}}^{2}\right)$, and $\xi_{\mathrm{GL}}=\sqrt{\pi D \hbar / 8\left(T_{c}-T\right)}$ is the Ginzburg-Landau correlation length.

\section{RESULTS}

\section{A. Diffusion-controlled flux flow}

When the temperature is sufficiently far from the critical one, the electron-phonon relaxation terms $v_{\text {out }} \tilde{f}_{L}$ and $j_{\text {in }}$ in the kinetic equation (31) can be neglected because they are much smaller than the diffusion one. Qualitatively, this approximation means that the nonequilibrium quasiparticles generated near the vortex can drift to infinity at a rate exceeding that of inelastic relaxation. This regime is called the diffusion-controlled flux flow, and it is realized in the temperature domain $\left(T_{c}-T\right) \tau_{\mathrm{ph}} \gg 1$. Below we analyze this scenario separately for different temperature intervals.

\section{Low-temperature limit}

At low temperatures, the sizable quasiparticle density exists only inside vortex cores where the superconducting order parameter is suppressed. In this case, it is sufficient to consider only the zero-energy GF for which parameter $\theta$ is purely imaginary, $\vartheta=0$. The dissipation is dominated by the charge relaxation processes described by the distribution function $f_{T}$. The effective temperature change described by the distortion of $f_{L}$ can be neglected. Then Eq. (30) can be written as follows:

$$
\nabla_{r}^{2} \tilde{f}_{T}-\left(\frac{1}{r^{2}}-\frac{2|\Delta|}{D} \sin \eta\right) \tilde{f}_{T}=\frac{|\Delta|}{D r} \sin \eta .
$$

As a result, the coefficients that determine FFC in Eq. (36) are given by

$$
\begin{aligned}
& \alpha=-\int_{0}^{\infty} r d r \partial_{r}|\Delta| \partial_{r} \sin \eta, \\
& \gamma=\int_{0}^{\infty} d r|\Delta|\left(2 \tilde{f}_{T}-1 / r\right) \sin \eta .
\end{aligned}
$$

Previously, the value of $\beta \approx 0.9$ was reported by GK [2]. The calculation was based on the approximate vortex solution taken from Ref. [3]. This vortex structure was obtained by solving iteratively the self-consistency equation (26). Each iteration step was performed as follows. First, for a given vortex profile the GFs at each Matsubara frequency were determined by solving the Usadel equation. Then these GFs were substituted into the self-consistency equation in order to calculate the updated order parameter distribution. The iteration procedure used in Ref. [3] started from the gap function, which is also known as the Clem ansatz [20]. However, instead of taking a sufficient number of iterations to reach self-consistency, only a single iteration step was performed in Ref. [3]. In this way the approximate vortex profile was obtained, which was used later to calculate the flux-flow conductivity at low fields [2].

To get the correct order parameter distribution we have performed a sufficient number of iterations to ensure that the order parameter changes with each update become negligible. With the help of the fully self-consistent vortex structure obtained in this way we found that $\beta=0.77$. The previously reported value of 0.9 is overestimated by $17 \%$. The disparity between the initial gap distribution and the one obtained after the first iteration and the exact gap function together with corresponding values of $\beta$ are shown in Fig. 1.

\section{High-temperature limit}

For elevated temperatures still in the diffusive-controlled limit $\left(T_{c}-T\right) \tau_{\mathrm{ph}} \gg 1$, the nonequilibrium states are dominated mostly by the change in the number of quasiparticles determined by the $f_{L}$ mode, while the charge imbalance $f_{T}$ yields the subdominant contribution. In this regime the FFC was calculated within the local-density approximation for the spectral functions $[4,6]$. This approximation results in expression (2) with $\beta_{0}=4.04$ (see Appendix D for the calculation details).

The local-density approximation is well justified in the limit $T \rightarrow T_{c}$. However, to stay in the diffusion-controlled regime the temperature cannot be taken infinitesimally close to the critical one. Thus, it is interesting to improve the accuracy of the $\beta$ calculation for small but finite values of $T_{c}-T$. For this purpose we found the order parameter solving the self-consistency equation (26) numerically. After that we considered Eq. (22) for the spectral functions, where the small parameter $1 / \tau$ regularizes the gap edge singularities. We fixed the value $\lambda_{\text {ph }} \sim 10^{-6}$ so that the relaxation time $\tau$ 

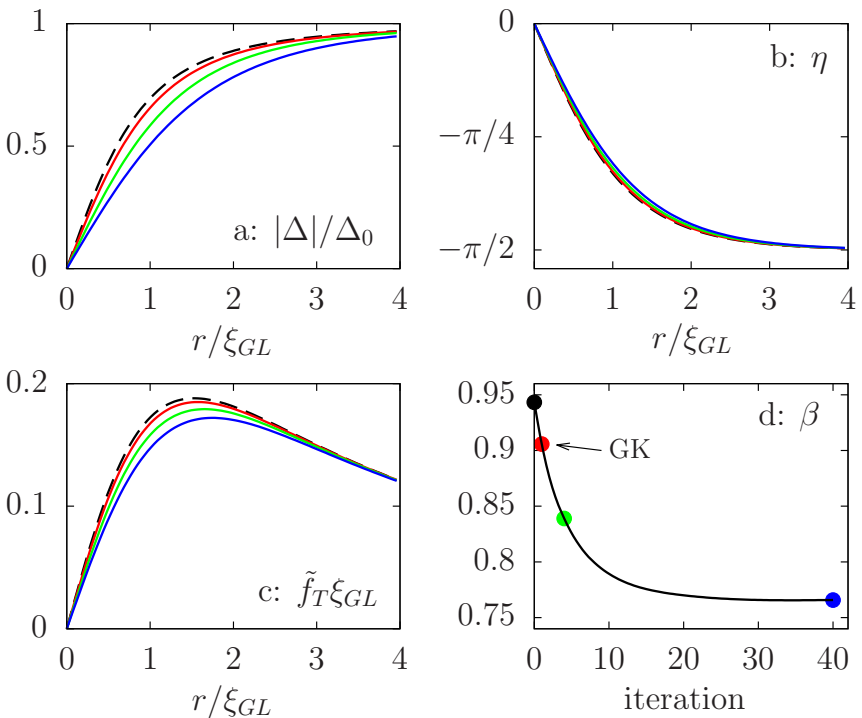

FIG. 1. (a) Single-vortex solution of the self-consistency equation solved by iterations at $T / T_{c}=0.05$. The initial-guess distribution given by the Clem ansatz (black dashed line) and the first iteration (red solid line) used in Ref. [2] are compared to the fourth (green solid line) and fortieth (blue solid line) iterations. (b) and (c) Values of the angle $\eta=\operatorname{Im} \theta$ and distribution function $\tilde{f}_{T}$ calculated based on gap profiles shown in (a). (d) The flux-flow conductivity slope $\beta$ depicted as a function of iteration number. Values of $\beta$ which correspond to the gap distributions shown in (a) are indicated by the dots with corresponding color. The notation GK refers to the result calculated by Gor'kov and Kopnin [2].

appears to be sufficiently large and diffusion-controlled FFC remains unaffected up to the temperatures $1-T / T_{c} \sim \lambda_{\text {ph. }}$. By starting with initial distributions for $\vartheta$ and $\eta$, we calculated the relaxation time $\tau$ according to Eq. (25) and then solved Eq. (22) numerically to get the new functions $\vartheta$ and $\eta$. By repeating this procedure iteratively, we found spectral functions with sufficient accuracy. By using these solutions, we calculated the relaxation rate $v_{\text {out }}$ for nonequilibrium longitudinal imbalance, Eq. (32), and solved the kinetic equations (30) and (31) by omitting the scattering-in term $j_{\text {in }}$.

Inclusion of finite relaxation time here is the technical trick needed to solve the equation for the $f_{L}$ mode at subgap energies numerically. Without relaxation, $f_{L}$ diverges exponentially as the function of the distance from the vortex core due to the conservation of the heat flow and exponential decay of the density of states far from the vortex. To find $f_{L}$ numerically, we remove its divergent behavior by electron-phonon relaxation. Note that FFC is not affected by the divergent asymptotic of $f_{L}$ since the latter is compensated by the decay of the density of states, e.g., in the expression for the force acting on the vortex (C6).

Figure 2 demonstrates the exactly calculated $\vartheta$ and $\eta$ and the distribution function $\tilde{f}_{L}$ compared to those obtained within the local-density approximation. With these functions we calculated integrals $\alpha$ and $\gamma$ [see expression (C7)]. As a result, we obtained the divergent behavior (2) with the dimensionless parameter $\beta_{0} \approx 3.7$. Therefore, the local-density approximation overestimates $\beta_{0}$ by $9 \%$.
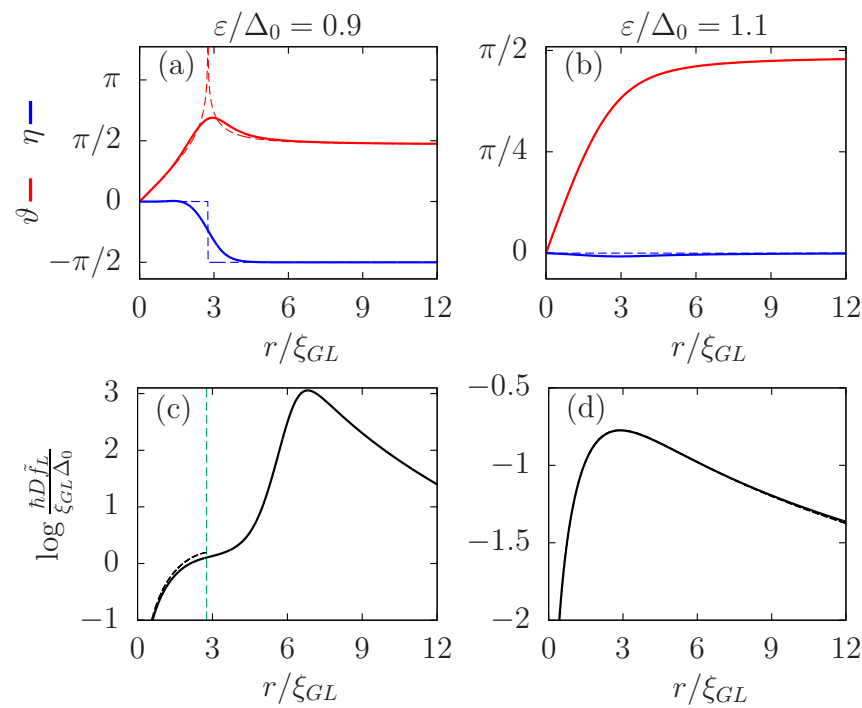

FIG. 2. $\vartheta=\operatorname{Re} \theta$ (red) and $\eta=\operatorname{Im} \theta$ (blue) as functions of distance for the energies below (a) and above (b) the bulk gap $\Delta_{0}$ and temperature $T / T_{c}=0.99$. Dashed curves represent solutions obtained within the local-density approximation used by LO. (c) and (d) The logarithm of $\tilde{f}_{L}$ (solid black) for the same energies. The dashed black curve in (c) corresponds to the distribution function used by LO [see Eq. (D1)]. The vertical line in (c) points to the distance where energy equals the local-density value, $\varepsilon=|\Delta|$. Note that for $\varepsilon>\Delta_{0}$ the difference between exact numerics and the local-density approximation is rather small.

\section{Intermediate temperatures}

For the temperatures within the broad range between limiting cases considered above, the contributions of both the $f_{L}$ and $f_{T}$ modes are generically of the same order of magnitude. Therefore, in order to calculate the FFC it is necessary to solve the system of coupled kinetic equations (30) and (31). This can be done only numerically, and the exact temperature dependence $\beta=\beta(T)$ in the diffusion-controlled regime has never been calculated before. Previously, only the interpolation curve between the GK and LO results was suggested [4]. Below we compare this interpolation curve with the result of an exact numerical calculation which is done in the same way as discussed in Sec. IV A 2 by repeating all steps at different temperatures.

Shown by the red curve in Fig. 3 is the obtained temperature dependence $\beta=\beta(T)$, which is qualitatively similar to the interpolation curve (black line) suggested previously in Ref. [4]. Both dependencies feature the gradual increase from the Bardeen-Stephen limit, $\beta \sim 1$, at small temperatures to the large values of $\beta$ at high temperatures due to the decrease in the diffusion relaxation rate. However, the calculated dependence $\beta(T)$ is significantly lower than the interpolation curve known from the literature.

\section{B. Effects due to inelastic relaxation}

Inelastic electron-phonon scattering provides an additional relaxation mechanism which affects FFC. This relaxation channel plays an important role at temperatures close to the critical one when the spatial gradients of the distribution 


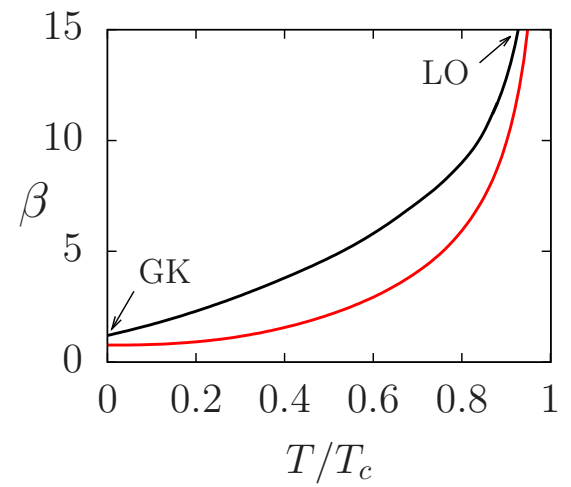

FIG. 3. Diffusion-controlled flux-flow conductivity parameter $\beta$ (36) as a function of temperature. The black line is the interpolation curve between GK and LO limits taken from Ref. [4] (their Fig. 13.1). The red line is a result of numerical calculation.

functions become small due to an increase in the correlation length and the superconducting energy gap is suppressed.

\section{Suppression of FFC}

The crossover between diffusion-controlled and inelastic relaxation-controlled branches of the $\beta(T)$ dependence occurs at temperatures $\tau_{\mathrm{ph}}\left(T_{c}-T\right) \sim 1$, where none of these approximations can be applied. The behavior of $\beta(T)$ in this region of parameters has not been studied before. To analyze the interplay between the two relaxation regimes we calculate numerically FFC for different inelastic scattering rates determined by the value of the parameter $\lambda_{\text {ph }}$. We apply the same numeric procedure as discussed in Sec. IV A 2.

Figure 4 shows the result of the calculation. Inelastic electron-phonon scattering suppresses the maximal value of FFC and smears the crossover from solely diffusion driven
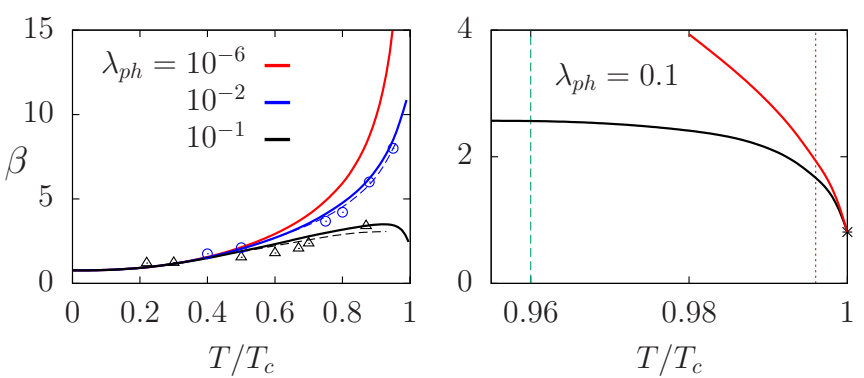

FIG. 4. Left: FFC as the electron-phonon relaxation rate determined by $\lambda_{\text {ph }}$ increases (solid curves). Experimental data are shown for $\mathrm{Zr}_{3} \mathrm{Rh}$ [14] (blue circles) and $\mathrm{Nb}_{0.5} \mathrm{Ta}_{0.5}$ [8,9] (black triangles). Dashed curves represent FFC calculated by means of distribution functions corrected by the scattering-in contribution to the collision integral (see text). Right: FFC calculated in the limit $T \rightarrow T_{c}$ by neglecting the nonequilibrium transverse imbalance mode (black curve). The red curve is the Tinkham contribution to FFC in the GTDGL theory, and the star corresponds to its limiting value, $\beta \approx 0.81$, in the gapless regime. Note that total FFC due to Tinkham and Bardeen-Stephen mechanisms is $\beta \approx 1.45$ at $T_{c}$. The dashed vertical line is defined by the condition $\hbar D / \xi_{\mathrm{GL}}^{2}=T_{c} \lambda_{\mathrm{ph}}$, and the dotted one is defined by $\hbar D / \xi_{\mathrm{GL}}^{2}=0.1 T_{c} \lambda_{\mathrm{ph}}$. to inelastic relaxation-controlled regimes. Such behavior is caused by suppressed generation of nonequilibrium quasiparticles due to the presence of the electron-phonon relaxation channel, so that nonequilibrium longitudinal imbalance becomes weaker. This follows from kinetic equation (31), where electron-phonon relaxation tends to suppress the source term determined by the density gradient.

To demonstrate the consistency of our numerics we first estimated the effect of the scattering-in contribution to the collision integral in kinetic equation (31). To do this we solved kinetic equations without the scattering-in term for the energies in the interval $\left[-20 \Delta_{0}, \ldots, 20 \Delta_{0}\right]$ and then calculated collision integral (33). By using its value we solved the kinetic equations again and obtained corrected distribution functions together with more accurate values of FFC, shown in the left panel of Fig. 4 by dashed curves. The effect of scattering-in term is rather small.

Next, we calculated FFC at elevated temperatures close to $T_{c}$. In this limit, we can compare our numerics with the results of GTDGL theory [21], which yields the decreasing behavior of $\beta$ with increasing temperature. The transition to this scenario is demonstrated by the lower curve in the left panel of Fig. 4, where $\beta$ passes through the maximum. However, our description is not very accurate in the limit of $T \rightarrow T_{c}$ because we do not take into account the order parameter modification by nonequilibrium effects. Such nonequilibrium self-consistency is needed to describe the screening of electric field by the Cooper pairs. Without including these effects, the contribution of the charge imbalance mode to FFC near $T_{c}$ becomes inaccurate. Therefore, to make a comparison with GTDGL theory we neglect the $f_{T}$ mode in kinetic equations (30) and (31) and the first term in the viscous friction force (C6). The remaining contribution to $\beta$ determined by the $f_{L}$ mode is shown in the right panel of Fig. 4. One can see that it gradually approaches the Tinkham contribution to $\beta$ obtained within the GTDGL model.

The right panel in Fig. 4 demonstrates also that the crossover towards the electron-phonon relaxation-controlled regime and the GTDGL theory takes place very close to the critical temperature where the electron-phonon relaxation rate is at least ten times larger than the one for the diffusion. The opposite condition $\hbar D / \xi_{\mathrm{GL}}=10 T_{c} \lambda_{\mathrm{ph}}$ is satisfied at the temperature $T / T_{c}=0.6$ for $\lambda_{\mathrm{ph}}=0.1$, and below this limit FFC is well approximated by only the diffusion mechanism (see Fig. 4). This suggests that the temperature interval where FFC is characterized by the coexistence of diffusion-driven and inelastic scattering-controlled mechanisms of relaxation can be quite wide, and neither of estimations (2) and (3) can give an adequate description in this region.

\section{Comparison with experiments}

The numerical value of $\lambda_{\mathrm{ph}}$ specific to the concrete superconductor can be directly obtained from the value of the conductivity parameter $\beta$ in the limit $T \rightarrow T_{c}$. However, for these large temperatures experimental studies of the vortex motion are challenging [7], and the behavior of FFC in this regime has not been studied in detail.

Above we demonstrated significant modifications of FFC caused by electron-phonon scattering, which allows us to 
obtain good numerical agreement with experimental data using the inelastic relaxation rate $\lambda_{\mathrm{ph}}=\left(\tau_{\mathrm{ph}} T_{c}\right)^{-1}$ as the fitting parameter. In real superconducting systems, FFC is strongly affected by electron-phonon relaxation so that applicability of Eqs. (2) and (3) appears to be very limited. In this case, the overall temperature behavior of FFC can be found only numerically due to the multicomponent mechanism of the nonequilibrium quasiparticle relaxation during the motion of the vortices.

In Fig. 4 we compare numerically calculated curves with experimental data for $\mathrm{Na}-\mathrm{Ta}$ system [8,9] and the amorphous superconductor $\mathrm{Zr}_{3} \mathrm{Rh}$ [14]. For the former case, a good fit is achieved for the value $\lambda_{\mathrm{ph}}=0.1$, which corresponds to the electron-phonon relaxation time of about $10^{-11} \mathrm{~s}$. This value agrees by the order of magnitude with ones reported previously for niobium [22,23]. For the system $\mathrm{Zr}_{3} \mathrm{Rh}$, good fit is found for $\lambda_{\mathrm{ph}}=0.01$ which corresponds to the electron-phonon relaxation time about $10^{-10} \mathrm{~s}$.

\section{SUMMARY}

To summarize, we have calculated the FFC in diffusive superconductors for small magnetic fields and arbitrary temperatures, taking into account the electron-phonon relaxation and using the self-consistent vortex solutions. First, we obtained the exact value of the dimensionless parameter $\beta=0.77$ which determines the FFC in the low-temperature limit $T \rightarrow 0$. Second, we calculated the overall temperature dependence of $\beta$ in the domain of the diffusion-controlled flux flow, that is, at $\tau_{\mathrm{ph}}\left(T_{c}-T\right) \gg 1$. Significant deviations from the previously reported interpolation curve are obtained. Finally, we studied the crossover between the diffusion-controlled and GTDGL regimes which occurs at $\tau_{\mathrm{ph}}\left(T_{c}-T\right) \sim 1$. The maximal value of $\beta$ obtained in this region is much smaller than expected from the estimations based on Eqs. (2) and (3) at the border of their applicability. Consequently, we obtained significant suppression of FFC near $T_{c}$ by changing the electron-phonon relaxation rate and achieved better agreement with the experimental data.

\section{ACKNOWLEDGMENTS}

This work was supported by the Estonian Ministry of Education and Research (Grant No. PUTJD141) and the Academy of Finland (Project No. 297439).

\section{APPENDIX A: DERIVATION OF KINETIC EQUATIONS}

The quasiclassical GF matrix defined in Eq. (4) obeys the Usadel equation

$$
\left\{\hat{\tau}_{3} \partial_{t}, \check{g}\right\}_{t}=D \hat{\partial}_{\mathbf{r}}\left(\check{g} \circ \hat{\partial}_{\mathbf{r}} \check{g}\right)+[\hat{H}, \check{g}]_{t}+\check{I},
$$

where $\hat{H}(\boldsymbol{r}, t)=i \hat{\Delta}-i e \phi \hat{\tau}_{0}, \hat{\Delta}(t)=i|\Delta| \hat{\tau}_{2} e^{-i \varphi \hat{\tau}_{3}}$ is the gap operator and $\check{I}=i(\check{g} \circ \check{\Sigma}-\check{\Sigma} \circ \check{g})$ is the collision integral due to relaxation processes described by the self-energy $\check{\Sigma}$. Equation (A1) is complemented by the normalization condition, and the parametrization of the Keldysh component is introduced in (8). Throughout the derivation we assume $k_{B}=\hbar=c=1$.
The diagonal elements of matrix equation (A1) give equations for $\hat{g}^{R / A}$ which have the same form as (A1) with $\hat{I}^{R / A}=i\left(\hat{g}^{R / A} \circ \hat{\Sigma}^{R / A}-\hat{\Sigma}^{R / A} \circ \hat{g}^{R / A}\right)$ substituted into it. The nondiagonal element reads

$$
\left\{\hat{\tau}_{3} \partial_{t}, \hat{g}^{K}\right\}_{t}=D \hat{\partial}_{\mathbf{r}}\left(\check{g} \circ \hat{\partial}_{\mathbf{r}} \check{g}\right)^{K}+\left[\hat{H}, \hat{g}^{K}\right]_{t}+\hat{I}^{K},
$$

where

$$
\begin{aligned}
\hat{I}^{K}= & i\left(\hat{g}^{R} \circ \hat{\Sigma}^{K}+\hat{g}^{K} \circ \hat{\Sigma}^{A}-\hat{\Sigma}^{R} \circ \hat{g}^{K}-\hat{\Sigma}^{K} \circ \hat{g}^{A}\right), \\
\hat{\partial}_{\mathbf{r}}\left(\check{g}^{\circ} \circ \hat{\partial}_{\mathbf{r}} \check{g}\right)^{K}= & \hat{\partial}_{\mathbf{r}}\left(\hat{g}^{R} \circ \hat{\partial}_{\mathbf{r}} \hat{g}^{K}+\hat{g}^{K} \circ \hat{\partial}_{\mathbf{r}} \hat{g}^{A}\right) \\
= & \hat{\partial}_{\mathbf{r}}\left(\hat{\partial}_{\mathbf{r}} \hat{f}-\hat{g}^{R} \circ \hat{\partial}_{\mathbf{r}} \hat{f} \circ \hat{g}^{A}\right)+\hat{g}^{R} \circ \hat{\partial}_{\mathbf{r}} \hat{g}^{R} \circ \hat{\partial}_{\mathbf{r}} \hat{f} \\
& -\hat{\partial}_{\mathbf{r}} \hat{f} \circ \hat{g}^{A} \circ \hat{\partial}_{\mathbf{r}} \hat{g}^{A}+\hat{\partial}_{\mathbf{r}}\left(\hat{g}^{R} \circ \hat{\partial}_{\mathbf{r}} \hat{g}^{R}\right) \circ \hat{f} \\
& -\hat{f} \circ \hat{\partial}_{\mathbf{r}}\left(\hat{g}^{A} \circ \hat{\partial}_{\mathbf{r}} \hat{g}^{A}\right) .
\end{aligned}
$$

To obtain last relation we substituted the parametrization (8) and used the associative property of differential superoperator $\hat{\partial}_{\mathbf{r}}\left(g_{1} \circ g_{2}\right)=\hat{\partial}_{\mathbf{r}} g_{1} \circ g_{2}+g_{1} \circ \hat{\partial}_{\mathbf{r}} g_{2}$. To get rid of the last two terms we subtract the spectral components of Eq. (A1) to obtain, finally, the equation

$$
\begin{aligned}
\hat{g}^{R} \circ & \left\{\hat{\tau}_{3} \partial_{t}, \hat{f}\right\}_{t}-\left\{\hat{\tau}_{3} \partial_{t}, \hat{f}\right\}_{t} \circ \hat{g}^{A} \\
= & D \hat{\partial}_{\boldsymbol{r}}\left(\hat{\partial}_{\boldsymbol{r}} \hat{f}-\hat{g}^{R} \circ \hat{\partial}_{\boldsymbol{r}} \hat{f} \circ \hat{g}^{A}\right) \\
& +D\left(\hat{g}^{R} \circ \hat{\partial}_{\mathbf{r}} \hat{g}^{R} \circ \hat{\partial}_{\boldsymbol{r}} \hat{f}-\hat{\partial}_{\boldsymbol{r}} \hat{f} \circ \hat{g}^{A} \circ \hat{\partial}_{\mathbf{r}} \hat{g}^{A}\right) \\
& +\hat{g}^{R} \circ[\hat{H}, \hat{f}]_{t}-[\hat{H}, \hat{f}]_{t} \circ \hat{g}^{A}+\hat{J},
\end{aligned}
$$

where $\hat{J}=\hat{Z} \circ \hat{g}^{A}-\hat{g}^{R} \circ \hat{Z}$ and $\hat{Z}=i\left(\hat{\Sigma}^{R} \circ \hat{f}-\hat{f} \circ \hat{\Sigma}^{A}-\right.$ $\left.\hat{\Sigma}^{K}\right)$. In our consideration, the collision integral describes only the electron-phonon scattering channel. This term is responsible for establishing equilibrium in the system.

To proceed we introduce the mixed representation in the time-energy domain as follows: $\hat{g}\left(t_{1}, t_{2}\right)=$ $\int_{-\infty}^{\infty} \hat{g}(\varepsilon, t) e^{-i \varepsilon\left(t_{1}-t_{2}\right)} \frac{d \varepsilon}{2 \pi}$, where $t=\left(t_{1}+t_{2}\right) / 2$. To keep the gauge invariance we introduce the modified GF $\hat{g}_{\text {new }}=$ $\hat{W}\left(t_{1}, t\right) \hat{g}\left(t_{1}, t_{2}\right) \hat{W}\left(t, t_{2}\right)$, where the link operator is defined in the text. This transformation removes the scalar potential term from the kinetic equations and adds the chemical potential shift $e \phi$. We absorb this shift by substituting $f_{T} \rightarrow f_{T}+e \phi \partial_{\varepsilon} f_{0}$, where $f_{0}(\varepsilon)=\tanh [\varepsilon /(2 T)]$ is the equilibrium distribution, so that $f_{T}$ hereafter denotes the deviation from the local equilibrium. Then keeping the first-order terms in frequency, we get the gradient approximation

$$
\begin{aligned}
{[\hat{H}, \hat{g}]_{t} } & =[\hat{H}, \hat{g}]-i\left\{\partial_{t} \hat{H}, \partial_{\varepsilon} \hat{g}\right\} / 2, \\
{\left[\boldsymbol{A} \hat{\tau}_{3}, \hat{g}\right]_{t} } & =\boldsymbol{A}\left[\hat{\tau}_{3}, \hat{g}\right]-i \partial_{t} \boldsymbol{A}\left\{\hat{\tau}_{3}, \partial_{\varepsilon} \hat{g}\right\} / 2, \\
\hat{\partial}_{\mathbf{r}} \hat{f}(\varepsilon, t) & =\nabla\left(f_{L} \hat{\tau}_{0}+f_{T} \hat{\tau}_{3}\right)+e \boldsymbol{E} \hat{\tau}_{3} \partial_{\varepsilon} f_{0},
\end{aligned}
$$

where $\boldsymbol{E}=-\nabla \phi-\partial_{t} \boldsymbol{A}$ is the electric field.

Here we assume first order in the deviation from equilibrium, so that the equilibrium distribution $f_{0}$ is substituted in the last term in (A5). With the same accuracy we obtain

$$
\begin{aligned}
& \hat{\partial}_{\mathbf{r}}\left(\hat{\partial}_{\mathbf{r}} \hat{f}-\hat{g}^{R} \circ \hat{\partial}_{\mathbf{r}} \hat{f} \circ \hat{g}^{A}\right) \\
& \quad=\hat{\nabla}\left(\hat{\nabla} \hat{f}_{1}-\hat{g}^{R} \hat{\nabla} \hat{f}_{1} \hat{g}^{A}\right)+e \partial_{\varepsilon} f_{0} \boldsymbol{\nabla} \cdot\left[\boldsymbol{E}\left(\hat{\tau}_{3}-\hat{g}^{R} \hat{\tau}_{3} \hat{g}^{A}\right)\right],
\end{aligned}
$$

where $\hat{f}_{1}=\left(f_{L}-f_{0}\right) \hat{\tau}_{0}+\hat{\tau}_{3} f_{T}$ is the deviation from the local equilibrium and $\hat{\nabla}=\nabla \hat{\tau}_{0}-i e \boldsymbol{A}\left[\hat{\tau}_{3},\right]$ is the gauge-covariant gradient. In (A6) we keep only terms which contribute to the kinetic equations. 
In the mixed representation the kinetic equation (A4) has the following gauge-invariant form:

$$
\begin{aligned}
& D \nabla\left(\nabla \hat{f}_{1}-\hat{g}^{R} \nabla \hat{f}_{1} \hat{g}^{A}\right)+D\left(\hat{g}^{R} \hat{\nabla} \hat{g}^{R} \nabla \hat{f}_{1}-\nabla \hat{f}_{1} \hat{g}^{A} \hat{\nabla} \hat{g}^{A}\right) \\
& \quad+\hat{g}^{R}\left[\hat{H}, \hat{f}_{1}\right]-\left[\hat{H}, \hat{f}_{1}\right] \hat{g}^{A}-i \partial_{\varepsilon} f_{0}\left(\hat{g}^{R} \hat{\partial}_{t} \hat{H}-\hat{\partial}_{t} \hat{H} \hat{g}^{A}\right) \\
& \quad+e D \partial_{\varepsilon} f_{0} \nabla \cdot\left[\boldsymbol{E}\left(\hat{\tau}_{3}-\hat{g}^{R} \hat{\tau}_{3} \hat{g}^{A}\right)\right] \\
& \quad+e D \partial_{\varepsilon} f_{0} \boldsymbol{E} \cdot\left(\hat{g}^{R} \hat{\nabla} \hat{g}^{R} \hat{\tau}_{3}-\hat{\tau}_{3} \hat{g}^{A} \hat{\nabla} \hat{g}^{A}\right)+\hat{J}=0 . \quad \text { (A7) }
\end{aligned}
$$

Here we took into account only first-order terms in the deviation from equilibrium and introduced the gauge-covariant time derivative $\hat{\partial}_{t}=\hat{\tau}_{0} \partial_{t}+2 i e \phi \hat{\tau}_{3}$.

To obtain Eqs. (10) and (11) in the main text we trace Eq. (A7) with Nambu matrices $\hat{\tau}_{0}$ and $\hat{\tau}_{3}$, respectively. Here we take into account that $\operatorname{Tr}\left(\hat{g}^{R} \hat{\tau}_{3} \hat{g}^{A}\right)=0$ because of the relation $\hat{g}^{A}=-\hat{\tau}_{3} \hat{g}^{R+} \hat{\tau}_{3}$ and the general form of the equilibrium spectral function $\hat{g}^{R}=g_{3} \hat{\tau}_{3}+g_{2} \hat{\tau}_{2} e^{-i \varphi \hat{\tau}_{3}}$. Then we neglect the driving terms with electric field and electron-phonon relaxation of the charge imbalance to get Eq. (10). We keep the electron-phonon collision integral in Eq. (11), which plays an important role in vortex dynamics being the only energy relaxation channel.

\section{APPENDIX B: COLLISION INTEGRALS}

We consider small nonstationary corrections to the GF in the form $\hat{g}^{R / A}=\hat{g}^{R / A}+\hat{g}_{\text {nst }}^{R / A}$ and $\hat{g}^{K}=\hat{g}^{R A} f_{0}+\hat{g}^{\text {nst }}$, where $\hat{g}^{R / A} \gg \hat{g}_{\text {nst }}^{R / A}$ and $\hat{g}^{\text {nst }}$ defined in Eq. (19). Here we use the notation $X^{R A}=X^{R}-X^{A}$ for $X=\hat{g}$. Then the stationary parts of the inelastic electron-phonon self-energy (15) read

$$
\begin{aligned}
\tilde{\Sigma}^{R / A}= & 2 i \omega|\omega|\left\{\hat{g}^{R / A}(\varepsilon+\omega)\left[\frac{1}{f_{0}(\omega)}-f_{0}(\varepsilon+\omega)\right]\right. \\
& \left.+\frac{f_{0}(\varepsilon+\omega)}{2}\left[\hat{g}^{R}(\varepsilon+\omega)+\hat{g}^{A}(\varepsilon+\omega)\right]\right\}, \\
\tilde{\Sigma}^{K}= & 2 i \omega|\omega| \hat{g}^{R A}(\varepsilon+\omega)\left[f_{0}(\varepsilon+\omega) / f_{0}(\omega)-1\right] .
\end{aligned}
$$

We are mostly interested in the self-energies at $\varepsilon \sim \Delta_{0}$, while the dominant contribution to the integral (15) comes from the region $\omega \gg \Delta_{0}$. Since for higher energies $\hat{g}^{R}+\hat{g}^{A} \ll \hat{g}^{R / A}$ and $\hat{g}^{R / A} \approx \hat{\tau}_{3} g^{R / A}$, the second contribution to $\tilde{\Sigma}^{R / A}$ in Eq. (B1) can be neglected, and the self-energy can be presented in the relaxation-time approximation, $i \hat{\Sigma}^{R / A}= \pm \hat{\tau}_{3} /(2 \tau)$, where $\tau$ is the energy-dependent inelastic electron-phonon collision time defined by

$$
\frac{1}{\tau}=\frac{\lambda_{\mathrm{ph}} \cosh \frac{\varepsilon}{2 T}}{14 \zeta(3) T_{c}^{2}} \int_{-\infty}^{\infty} \frac{\omega|\omega| d \omega g^{R}(\varepsilon+\omega)}{\sinh \frac{\omega}{2 T} \cosh \frac{\varepsilon+\omega}{2 T}} .
$$

This expression coincides with the formula used by WattsTobin et al. [21]. In Eq. (B2), relaxation time $\tau$ can contain an imaginary part due to complex $g^{R}$. Usually, this contribution is absorbed by renormalizing the chemical potential. Note that near the critical temperature, where $g^{R} \approx 1$ and $\varepsilon \ll \omega \sim T_{c}$, the inelastic electron-phonon collision time approaches the value $\tau_{\mathrm{ph}}=1 /\left(\lambda_{\mathrm{ph}} T_{c}\right)$.

Next, we express nonstationary contributions to selfenergies via $\hat{g}_{\text {nst }}^{R / A}$ and $\hat{g}^{\text {nst }}$ and derive the mixed representation for $\hat{Z}=i\left(\hat{\Sigma}^{R} \circ \hat{f}-\hat{f} \circ \hat{\Sigma}^{A}-\hat{\Sigma}^{K}\right)$. The latter quantity does not contain stationary terms. For the collision integral $\hat{J}=\hat{Z}$ 。 $\hat{g}^{A}-\hat{g}^{R} \circ \hat{Z}$ in the mixed representation we obtain with the help of the GF in Nambu space $\operatorname{Tr} \hat{J}=-\left(f_{L}-f_{0}\right) v_{\text {out }}+J_{\text {in }}$, where

$$
\begin{aligned}
v_{\mathrm{out}}= & \frac{\lambda_{\mathrm{ph}}}{28 \zeta(3) T_{c}^{2}} \int_{-\infty}^{\infty} d \omega \omega|\omega|\left\{2 g^{R A}(\varepsilon) g^{R A}(\varepsilon+\omega)\right. \\
& \left.-f^{R A}(\varepsilon) f^{R+A+}(\varepsilon+\omega)-f^{R+A+}(\varepsilon) f^{R A}(\varepsilon+\omega)\right\} \\
& \times\left[1 / f_{0}(\omega)-f_{0}(\varepsilon+\omega)\right], \\
J_{\text {in }}= & \frac{\lambda_{\mathrm{ph}}}{28 \zeta(3) T_{c}^{2}} \int_{-\infty}^{\infty} d \omega \omega|\omega|\left\{2 g^{R A}(\varepsilon) g^{R A}(\varepsilon+\omega)\right. \\
& \left.-f^{R A}(\varepsilon) f^{R+A+}(\varepsilon+\omega)-f^{R+A+}(\varepsilon) f^{R A}(\varepsilon+\omega)\right\} \\
& \left.\times\left[f_{L}(\varepsilon+\omega)-f_{0}(\varepsilon+\omega)\right]\left[f_{0}(\varepsilon)+1 / f_{0}(\omega)\right] . \quad \text { B } 4\right)
\end{aligned}
$$

Here we used the notation $f^{R+A+}=f^{R+}-f^{A+}$. In expressions (B3) and (B4) the dominant contribution to the integrals comes from high energies. Since $f_{L}-f_{0}$ is significant only at low energies, the scattering-in term $J_{\text {in }}$ appears to be a small correction to the collision integral. Note that $\nu_{\text {out }} \rightarrow 2 g^{R A} / \tau$ if the temperature approaches the critical one.

\section{APPENDIX C: $\theta$ PARAMETRIZATION}

The Usadel equation for equilibrium spectral functions has the form

$$
D \hat{\partial}_{\mathbf{r}}\left(\hat{g}^{R / A} \hat{\partial}_{\mathbf{r}} \hat{g}^{R / A}\right)+\left[i \varepsilon \hat{\tau}_{3}+i \hat{\Delta}-i \hat{\Sigma}^{R / A}, \hat{g}^{R / A}\right]=0 .
$$

By deriving this equation, we took into account that in the mixed representation $\left\{\hat{\tau}_{3} \partial_{t}, \hat{g}\right\}_{t}=-i \varepsilon\left[\hat{\tau}_{3}, \hat{g}\right]$ and the scalar potential is neglected at equilibrium.

By using parametrizations (20) and (21) one finds in cylindrical coordinates

$$
\begin{aligned}
& {\left[\hat{\Delta}+\varepsilon \hat{\tau}_{3}+i \hat{\tau}_{3} /(2 \tau), \hat{g}^{R}\right]} \\
& =2\{[\varepsilon+i /(2 \tau)] \sinh \theta-|\Delta| \cosh \theta\} \hat{\tau}_{1} e^{-i \hat{\tau}_{3} \varphi}, \\
& \quad \nabla\left(\hat{g}^{R} \nabla \hat{g}^{R}\right)=\left[\nabla_{r}^{2} \theta-\sinh (2 \theta) /\left(2 r^{2}\right)\right] \hat{\tau}_{1} e^{-i \hat{\tau}_{3} \varphi},
\end{aligned}
$$

where $\nabla_{r}^{2}=\partial_{r}^{2}+r^{-1} \partial_{r}$. By taking into account that selfenergy $\hat{\Sigma}^{R / A}$ in Eq. (C1) corresponds to the stationary contribution, $2 i \hat{\Sigma}^{R / A}= \pm \hat{\tau}_{3} / \tau$ (see Appendix B), we obtain Eq. (22) for $\theta(r)$.

It is convenient to split $\theta$ into the real and imaginary parts, $\vartheta=\operatorname{Re} \theta$ and $\eta=\operatorname{Im} \theta$, which satisfy the following equations:

$$
\begin{aligned}
& \nabla_{r}^{2} \vartheta-\frac{\sinh (2 \vartheta) \cos (2 \eta)}{2 r^{2}}=\frac{2}{D}\left(\varepsilon \cosh \vartheta \sin \eta+\frac{1}{2 \tau} \sinh \vartheta \cos \eta-|\Delta| \sinh \vartheta \sin \eta\right), \\
& \nabla_{r}^{2} \eta-\frac{\cosh (2 \vartheta) \sin (2 \eta)}{2 r^{2}}=\frac{2}{D}\left(|\Delta| \cosh \vartheta \cos \eta-\varepsilon \sinh \vartheta \cos \eta+\frac{1}{2 \tau} \cosh \vartheta \sin \eta\right),
\end{aligned}
$$

supplemented by the boundary conditions (23). 
With the help of parametrizations (20) and (21), the kinetic equation can be simplified due to the following identities:

$$
\begin{aligned}
& \mathcal{D}_{T}=2 D[1+\cosh (2 \vartheta)], \\
& 2 i \operatorname{Tr}\left[\left(\hat{g}^{R}+\hat{g}^{A}\right) \hat{\Delta}\right]=8|\Delta| \cosh \vartheta \sin \eta, \\
& \operatorname{Tr}\left[\hat{\tau}_{3} \partial_{t} \hat{\Delta}\left(\hat{g}^{R}+\hat{g}^{A}\right)\right]=-4\left(\boldsymbol{v}_{L} \cdot \nabla \varphi\right)|\Delta| \cosh \vartheta \sin \eta, \\
& \mathcal{D}_{L}=2 D[1+\cos (2 \eta)], \\
& \operatorname{Tr}\left[\hat{\tau}_{3}\left(\hat{g}^{R}-\hat{g}^{A}\right) \hat{\Delta}\right]=0, \\
& \operatorname{Tr}\left[\partial_{t} \hat{\Delta}\left(\hat{g}^{R}-\hat{g}^{A}\right)\right]=4\left(\boldsymbol{v}_{L} \cdot \nabla|\Delta|\right) \sinh \vartheta \cos \eta, \\
& \boldsymbol{j}_{e}=-2 D \sinh (2 \vartheta) \sin (2 \eta) \nabla \varphi,
\end{aligned}
$$

where we took into account that for the vortex moving with constant velocity $\partial_{t} \Delta=-\boldsymbol{v}_{L} \cdot \nabla \Delta$. By construction, the spectral current is conserved, $\nabla \cdot j_{e}=0$. Taking into account that $\boldsymbol{v}_{L} \cdot \nabla \varphi=-v_{L} \sin \varphi / r$ and $\boldsymbol{v}_{L} \cdot \nabla|\Delta|=v_{L} \cos \varphi \partial_{r}|\Delta|$, we arrive at Eqs. (30) and (31), where the collision integral $\operatorname{Tr} \hat{J}=-\left(f_{L}-f_{0}\right) v_{\text {out }}+J_{\text {in }}$ (see Appendix B) is substituted. After that, we used the $\theta$ parametrization to obtain

$$
2 g^{R A}(\varepsilon) g^{R A}\left(\varepsilon^{\prime}\right)-f^{R A}(\varepsilon) f^{R+A+}\left(\varepsilon^{\prime}\right)-f^{R+A+}(\varepsilon) f^{R A}\left(\varepsilon^{\prime}\right)=8 \cos [\eta(\varepsilon)] \cos \left[\eta\left(\varepsilon^{\prime}\right)\right] \cosh \left[\vartheta(\varepsilon)-\vartheta\left(\varepsilon^{\prime}\right)\right]
$$

and renormalized the scattering-in part, namely, $J_{\text {in }}=v_{L} j_{\text {in }} \cos \varphi \partial_{\varepsilon} f_{0}$.

To calculate the force $\boldsymbol{F}_{\text {env }}$ (18) we use the expansion (19) and the spectral functions in the form (20) and (21). Using the ansatz (28), we get an expression for the force in the form

$$
\begin{aligned}
\boldsymbol{F}_{e n v}= & \frac{\nu v_{L}}{2} \int d^{2} \boldsymbol{r} d \varepsilon \partial_{\varepsilon} f_{0}\left\{\sin \varphi \cosh \vartheta \sin \eta|\Delta|\left(2 \tilde{f}_{T}-1 / r\right) \nabla \varphi\right. \\
& \left.+\cos \varphi\left[\partial_{r}(\cosh \vartheta \sin \eta)-2 \tilde{f}_{L} \sinh \vartheta \cos \eta\right] \nabla|\Delta|\right\} .
\end{aligned}
$$

After integration, this can be written as $\boldsymbol{F}_{\text {env }}=-\varrho \boldsymbol{v}_{L}$, where the viscosity coefficient is given by $\varrho=\pi \hbar \nu(\alpha+\gamma)$ and

$$
\begin{aligned}
& \alpha=\int_{0}^{\infty} r d r \partial_{r}|\Delta| \int_{0}^{\infty} d \varepsilon \partial_{\varepsilon} f_{0}\left[2 \tilde{f}_{L} \sinh \vartheta \cos \eta-\partial_{r}(\cosh \vartheta \sin \eta)\right], \\
& \gamma=\int_{0}^{\infty} d r|\Delta| \int_{0}^{\infty} d \varepsilon \partial_{\varepsilon} f_{0} \cosh \vartheta \sin \eta\left(2 \tilde{f}_{T}-1 / r\right) .
\end{aligned}
$$

Here we have taken into account that $\tilde{f}_{T}$ and $\eta$ are even, while $\tilde{f}_{L}$ and $\vartheta$ are odd functions of energy $\varepsilon$.

\section{APPENDIX D: DERIVATION OF THE LO RESULT}

Following LO [6], the analytical result for diffusion-driven FFC can be obtained by noticing that near $T_{c}$ the diffusion terms in the Usadel equation (22) are much smaller than the gap field. As a result, the local-density approximation can be implemented, where $\vartheta$ and $\eta$ are determined by their homogeneous expressions with bulk gap substituted by the local value of gap field.

To calculate conductivity contributions (C7), it is convenient to consider energetic integration in the domains $[0, \ldots,|\Delta(r)|]$ and $[|\Delta(r)|, \ldots, \infty]$ separately. The former gives a negligible contribution close to $T_{c}$ and can be omitted. In the latter case, the energetic integration variable exceeds the local gap value, and local approximation results in $\eta=0$ and $\sinh \vartheta=|\Delta(r)| / \sqrt{\varepsilon^{2}-|\Delta(r)|^{2}}$. In this case, $\tilde{f}_{T}=\gamma=0$, and kinetic equation (31) is satisfied by the solution [6]

$$
\tilde{f}_{L}=\frac{1}{r \hbar D} \int_{0}^{r} d r_{1} r_{1}\left[\sqrt{\varepsilon^{2}-|\Delta|^{2}}-C(\varepsilon)\right] .
$$

The condition for the vanishing heat current $\partial_{r} \tilde{f}_{L}=0$ in the bulk defines the constant $C=\sqrt{\varepsilon^{2}-\Delta_{0}^{2}} \equiv C_{1}$ at large energies $\varepsilon>\Delta_{0}$. For the subgap region $\varepsilon<\Delta_{0}$, LO used a boundary condition with zero heat current at the interface $r=r_{\varepsilon}$ defined by $\varepsilon=\left|\Delta\left(r_{\varepsilon}\right)\right|$. This determines the integration constant for $\varepsilon<\Delta_{0}$ in the form $C=-\frac{2}{r^{2}} \int_{0}^{r_{\varepsilon}} r d r \sqrt{\varepsilon^{2}-|\Delta(r)|^{2}} \equiv C_{2}$.

The dominant contribution to viscosity (C7) stems from integration over the $\varepsilon$ - $r$ domain enclosed by $r=0$ and $\varepsilon=$ $|\Delta(r)|$ curves. One obtains $\alpha=\alpha_{1}+\alpha_{2}$, where

$$
\begin{aligned}
& \alpha_{1}=\frac{2}{\hbar D} \int_{\Delta_{0}}^{\infty} d \varepsilon \partial_{\varepsilon} f_{0} \int_{0}^{\infty} d r r\left[\sqrt{\varepsilon^{2}-|\Delta|^{2}}-C_{1}(\varepsilon)\right]^{2}, \\
& \alpha_{2}=\frac{2}{\hbar D} \int_{0}^{\Delta_{0}} d \varepsilon \partial_{\varepsilon} f_{0}\left[\int_{0}^{r_{\varepsilon}} d r r\left(\varepsilon^{2}-|\Delta|^{2}\right)+\frac{r_{\varepsilon}^{2}}{2} C_{2}(\varepsilon)^{2}\right] .
\end{aligned}
$$

By finding the gap profile near $T_{c}$ numerically, we calculated these integrals and obtained $\alpha_{1,2} \approx(0.409$; $0.496) \xi_{\mathrm{GL}}^{2} \Delta_{0}^{3} /\left(\hbar D T_{c}\right)$. As a result, $\beta=\beta_{0} \sqrt{T_{c} /\left(T_{c}-T\right)}$, where $\beta_{0}=4.01$. 
[1] J. Bardeen and M. J. Stephen, Phys. Rev. 140, A1197 (1965).

[2] L. P. Gor'kov and N. B. Kopnin, Sov. Phys. JETP 38, 195 (1974).

[3] R. J. Watts-Tobin and G. M. Waterworth, in Low Temperature Physics-LT 13: Superconductivity, edited by K. D. Timmerbaus, W. J. O’Sullivan, and E. F. Hammel (Plenum, New York, 1974), p. 37.

[4] N. Kopnin, Theory of Nonequilibrium Superconductivity (Oxford University Press, Oxford, 2001).

[5] L. P. Gor'kov and N. B. Kopnin, Sov. Phys. JETP 37, 183 (1973).

[6] A. I. Larkin and Yu. N. Ovchinnikov, Sov. Phys. JETP 46, 155 (1977).

[7] L. P. Gor'kov and N. B. Kopnin, Sov. Phys. Usp. 18, 496 (1975).

[8] Y. B. Kim, C. F. Hempstead, and A. R. Strnad, Phys. Rev. 139, A1163 (1965).

[9] W. F. Vinen and A. C. Warren, Proc. Phys. Soc. 91, 399 (1967).

[10] I. N. Goncharov, G. L. Dorofeev, A. Nichitiu, L. V. Petrova, D. Fricsovszky, and I. S. Khukhareva, Sov. Phys. JETP 40, 1109 (1975).

[11] Y. Muto, K. Mori, and K. Noto, Physica 55, 362 (1971).
[12] T. Takayama, J. Low Temp. Phys. 27, 359 (1977).

[13] N. Y. Fogel', Sov. Phys. JETP 36, 725 (1973).

[14] S. J. Poon and K. M. Wong, Phys. Rev. B 27, 6985 (1983).

[15] A. Schmid, Phys. Kondens. Mater. 5, 302 (1966).

[16] G. M. Eliashberg, Sov. Phys. JETP 34, 668 (1972).

[17] A. I. Larkin and Y. N. Ovchinnikov, in Modern Problems in Condensed Matter Sciences: Nonequilibrium Superconductivity, edited by D. N. Langenberg and A. Larkin (Elsevier, New York, 1986), p. 493.

[18] M. Silaev and A. Vargunin, Phys. Rev. B 94, 224506 (2016).

[19] K. Maki, J. Low Temp. Phys. 1, 45 (1969).

[20] J. R. Clem, J. Low Temp. Phys. 18, 427 (1975).

[21] R. J. Watts-Tobin, J. Low Temp. Phys. 42, 459 (1981).

[22] Y. P. Gousev, G. N. Gol'tsman, A. D. Semenov, E. M. Gershenzon, R. S. Nebosis, M. A. Heusinger, and K. F. Renk, J. Appl. Phys. 75, 3695 (1994).

[23] N. G. Ptitsina, G. M. Chulkova, K. S. Ilin, A. V. Sergeev, F. S. Pochinkov, E. M. Gershenzon, and M. E. Gershenson, Phys. Rev. B 56, 10089 (1997). 